\title{
Different chromosome damage in lymphocytes of newly diagnosed gastrointestinal and breast cancer patients
}

\author{
D. KADLCIKOVA ${ }^{1}$, P. MUSILOVA ${ }^{1, *}$, H. HRADSKA ${ }^{1}$, M. PETROVOVA ${ }^{2}$, I. SELINGEROVA ${ }^{3}$, M. VOZDOVA ${ }^{1}$, M. SVOBODA ${ }^{2}$, J. RUBES ${ }^{1}$
}

${ }^{1}$ Department of Genetics and Reproduction, Central European Institute of Technology - Veterinary Research Institute, Brno, Czech Republic; ${ }^{2}$ Clinic of Comprehensive Cancer Care, Masaryk Memorial Cancer Institute, Brno, Czech Republic, ${ }^{3}$ Regional Centre for Applied Molecular Oncology, Masaryk Memorial Cancer Institute, Brno, Czech Republic

${ }^{*}$ Correspondence: musilova@vri.cz

Received June 4, 2019 /Accepted September 16, 2019

\begin{abstract}
Structural chromosome aberrations are a predictive biomarker of cancer risk. Conventional chromosome analysis widely used for these purposes detects unstable chromosome aberrations that are eliminated during cell division. Stable aberrations that may persist in the body and tend to accumulate during a lifetime can be detected by fluorescence in situ hybridization (FISH). The aim of the study was to investigate the level of chromosome damage in newly diagnosed cancer patients and control subjects by FISH. Both groups of untreated cancer patients had increased frequency of aberrant cells. However, chromosome damage affected different cytogenetic endpoints. Stable translocations and cells with complex rearrangements were elevated in breast cancer patients whereas unstable chromosome aberrations (dicentric chromosomes and acentric fragments) were elevated in gastrointestinal cancer patients. These associations observed in nonsmokers were typically not pronounced in smokers (with the exception of dicentric chromosomes in gastrointestinal patients). Exposure to tobacco smoke increased aberrations in healthy controls but not in the cancer patients. Our study suggests an association between cancer and stable chromosomal rearrangements in breast cancer patients. Unstable aberrations elevated in gastrointestinal cancer patients may be at least partly ascribed to the exposure to diagnostic X-rays.
\end{abstract}

Key words: breast cancer, gastrointestinal cancer, risk assessment, chromosome aberration, fluorescence in situ hybridization

Genomic instability, including changes in chromosome number and structure, is a typical feature of neoplastic cells. Therefore, using chromosome abnormalities as a possible biomarker to indicate cancer risk was verified by many studies. It has been shown that increased frequency of chromosome damage in healthy individuals indicates an increased cancer risk with the strongest association found for stomach cancer [1-3]. Several studies also investigated chromosome aberrations in newly diagnosed, untreated cancer patients. Association with tumor development was suggested in lung, breast, and colorectal cancer, as well as Hodgkin's lymphoma [4-8].

Of the cytogenetic endpoints, structural chromosome aberrations are the most robust predictive biomarker of cancer risk [9]. Conventional chromosome analysis using Giemsa stained metaphase chromosomes is widely used for these purposes. This method detects unstable chromosome aberrations, such as chromosome or chromatid breaks and dicentric chromosomes. However, the number of unstable aberrations decreases with time, as they are lost through cell divisions [10]. Thus, it mostly exposes recently occur- ring chromosome damage. Scoring of dicentric chromosomes is suitable for biological dosimetry of recent and acute exposures to ionizing radiation $[11,12]$.

In addition to unstable chromosome aberrations, the fluorescence in situ hybridization (FISH) technique using painting probes is also able to detect chromosome aberrations that can survive mitotic division. Stable aberrations may persist in the body for years and tend to accumulate during a lifetime. Therefore, translocation analysis is used as a biomarker for evaluating the received doses of ionizing radiation from past or chronic exposure [13]. FISH seems to be more sensitive than conventional chromosome analysis as was shown in medical personnel and in the study of environmental air pollution impact [14-17]. Nevertheless, only a limited number of studies have used FISH to study the association between chromosome aberrations and cancer risk.

Using FISH with painting probes, we investigated the level of chromosome damage in peripheral blood lymphocytes taken from a group of patients who were newly diagnosed 
with gastrointestinal and breast cancer compared to healthy control subjects. The aim of our study was to validate chromosome aberrations detected by FISH as a predictive biomarker of cancer risk.

\section{Patients and methods}

Subjects (participants). In total, we investigated 124 patients with newly diagnosed solid tumors from Masaryk Memorial Cancer Institute, Brno (MMCI). The group comprised 27 patients (16 males and 11 females; 3 current smokers, 11 former smokers and 13 nonsmokers) with gastrointestinal cancer (cancer of the esophagus, stomach, intestine, colon, rectum, liver and pancreas) and 97 female patients with breast cancer (14 current smokers, 16 former smokers and 67 nonsmokers). According to the TNM (tumor, node, metastasis) system, gastrointestinal cancer patients were in stages: I (8), II (8), III (8) and IV (3). Most of the breast cancer patients were in stages I (54) and II (40), only 2 patients were in stage III and 1 patient was not classified. Blood samples were taken from the patients prior to cancer treatment (radiotherapy or chemotherapy). A group of 79 healthy control subjects ( 37 males and 42 females; 8 current smokers, 27 former smokers and 44 nonsmokers) was recruited through the outpatient clinic of preventive oncology at the MMCI who have not suffered from cancer and live and work in a nonhazardous environment.

All participants provided informed consent for their participation in the study and completed a questionnaire. Information about lifestyle, potential occupational exposure, other diseases, and their treatments were collected. All acquired data and blood samples were coded and processed anonymously.

Culture of blood. Cultures from samples of heparinized venous blood were established in complete Chromosome $\mathrm{P}$ Medium (Euroclone, Pero, Italy) and incubated at $37^{\circ} \mathrm{C}$ for 48 h. Colcemid (Sigma-Aldrich, St. Louis, MO, USA) was added to a final concentration of $0.1 \mu \mathrm{g} / \mathrm{ml} 2 \mathrm{~h}$ before the end of incubation. The culture harvest was made using a $20 \mathrm{~min}$ hypotonic treatment in $0.075 \mathrm{M} \mathrm{KCl}$ prewarmed to $37^{\circ} \mathrm{C}$ and (3:1) methanol : acetic acid fixation. Metaphase chromosome spreads were prepared by dropping the cell suspensions onto moistened microscopic slides, which were then air-dried.

FISH. Painting probes for chromosomes 1, 2 and 4 were used because of their ability to visualize a large fraction of genome. Prior to hybridization, the locations of DAPI (4, 6-diamidino-2-phenylindole) stained metaphase cells, potentially suitable for further analysis, were established by an automatic metaphase finder system (Metafer) and the coordinates were saved. The slides were denatured in $0.07 \mathrm{M}$ $\mathrm{NaOH}$ (according to a protocol provided by MetaSystems for multi-color probe kits) and hybridized with denatured painting probes for chromosomes 1 (orange), 2 (green) and 4 (green; MetaSystems) and a pancentromeric probe (biotin; Cambio). After overnight hybridization at $37^{\circ} \mathrm{C}$, the slides were washed in $0.4 \times$ SSC at $72^{\circ} \mathrm{C}$ and biotin labeled probe was detected by avidin conjugated to the near infrared fluorescent dye Cy5. Chromosomes were counterstained with DAPI. The metaphase spreads were relocated by Metafer, and 1000 cells from each sample were scored under a fluorescence microscope equipped with dual band excitation filter set for green and orange fluorescence and filters for single dyes including DAPI and Cy5. Aberrant or potentially aberrant cells were captured and classified by two independent evaluators using image analysis software ISIS (MetaSystems) according to the Protocol for Aberration Identification and Nomenclature [18]. Reciprocal translocations, the total number of translocations (translocated chromosomes), dicentric chromosomes, acentric fragments, and cells with complex rearrangements were evaluated. To calculate whole genome translocation frequency each complete reciprocal translocation (2-way translocations) and incomplete translocation (1-way translocations) were considered as one event. Apparently stable complex exchanges ( 3 or more breaks in 2 or more chromosomes) were broken down into simple translocations and included in the total translocation count [19, 20]. Genomic frequencies of stable chromosomal translocations $\left(\mathrm{F}_{\mathrm{G}}\right)$ were calculated according to a standard formula proposed by Lucas and Sachs [21].

Statistical evaluation of data. Data were analyzed using the statistical program $\mathrm{R}$ version 3.3.3 (https://www.Rproject.org). All tests were performed at a significance level of 5\%. The Mann-Whitney test was used to compare distributions of individual parameters between groups. To control the confounding effects of age and smoking, binary logistic regression was used and adjusted odds ratios (aOR) with $95 \%$ confidence intervals were calculated.

\section{Results}

Using FISH, we investigated chromosomal aberrations in 124 patients with newly diagnosed solid tumors (median age 62.6 years) and 79 healthy control individuals (median age 54.2 years). The group of patients included 27 with gastrointestinal cancer (median age 63.4 years) and 97 with breast cancer (median age 62.3 years). The detailed results of the cytogenetic investigation are summarized in Table 1 . Higher frequencies of aberrant cells were observed in gastrointestinal cancer (median 14/1000 cells, $\mathrm{p}=0.001$ ) and breast cancer (median $10 / 1000$ cells, $\mathrm{p}=0.026$ ) patients than in controls (median 8/1000 cells). However, the groups of gastrointestinal cancer and breast cancer patients were older than controls ( $p=0.009$ and $p=0.007$, respectively) and the proportion of smokers in patients differed from the proportion in controls. Therefore, the association between cytogenetic damage and cancer development (expressed as odds ratios) was adjusted for two main confounders - age and smoking. The results are summarized in Table 2 . The analysis revealed significantly increased frequencies of aberrant cells $(\mathrm{aOR}=1.16, \mathrm{p}=0.009)$, dicentric chromosomes $(\mathrm{aOR}=2.70$, 
Table 1. Chromosomal aberrations in newly diagnosed cancer patients and controls.

\begin{tabular}{|c|c|c|c|c|c|}
\hline & & \multicolumn{3}{|c|}{ Cancer patients } & \multirow[b]{2}{*}{$\begin{array}{c}\text { Controls } \\
\mathrm{N}=79\end{array}$} \\
\hline & & $\begin{array}{c}\text { Gastrointestinal } \\
\mathrm{N}=\mathbf{2 7}\end{array}$ & $\begin{array}{l}\text { Breast } \\
\mathrm{N}=97\end{array}$ & $\begin{array}{c}\text { Total } \\
\mathrm{N}=124\end{array}$ & \\
\hline Age & mean $\pm \mathrm{SD}$ & $61.9 \pm 9.8$ & $60.4 \pm 12.3$ & $60.7 \pm 11.8$ & $54.8 \pm 12.9$ \\
\hline \multirow[t]{3}{*}{ (years) } & median & 63.4 & 62.3 & 62.6 & 54.2 \\
\hline & first quartile & 54.8 & 51.0 & 51.5 & 45.5 \\
\hline & third quartile & 69.0 & 68.6 & 68.8 & 65.8 \\
\hline \multirow[t]{4}{*}{ Ab.c. } & mean $\pm S D$ & $13.0 \pm 6.0$ & $10.3 \pm 5.1$ & $10.9 \pm 5.4$ & $8.7 \pm 4.4$ \\
\hline & median & 14 & 10 & 10.5 & 8 \\
\hline & first quartile & 7 & 7 & 7 & 5 \\
\hline & third quartile & 17 & 14 & 14 & 11 \\
\hline \multirow[t]{4}{*}{$\mathrm{rcp}$} & mean $\pm \mathrm{SD}$ & $7.2 \pm 4.4$ & $6.7 \pm 4.1$ & $6.8 \pm 4.1$ & $5.5 \pm 3.7$ \\
\hline & median & 7 & 5 & 6 & 5 \\
\hline & first quartile & 4 & 4 & 4 & 3 \\
\hline & third quartile & 11 & 9 & 10 & 8 \\
\hline \multirow[t]{4}{*}{$\mathrm{t}$} & mean $\pm S D$ & $18.1 \pm 10.2$ & $16.5 \pm 9.1$ & $16.8 \pm 9.3$ & $13.3 \pm 8.4$ \\
\hline & median & 18 & 14 & 15.5 & 12 \\
\hline & first quartile & 9 & 9 & 9 & 7 \\
\hline & third quartile & 25 & 22 & 23 & 18 \\
\hline \multirow[t]{4}{*}{ dic } & mean $\pm \mathrm{SD}$ & $2.1 \pm 1.4$ & $1.0 \pm 1.2$ & $1.3 \pm 1.3$ & $0.8 \pm 0.9$ \\
\hline & median & 2 & 1 & 1 & 1 \\
\hline & first quartile & 1 & 0 & 0 & 0 \\
\hline & third quartile & 3 & 2 & 2 & 1 \\
\hline \multirow[t]{4}{*}{ ace } & mean $\pm \mathrm{SD}$ & $5.3 \pm 3.6$ & $2.8 \pm 2.6$ & $3.4 \pm 3.0$ & $2.5 \pm 2.4$ \\
\hline & median & 4 & 2 & 3 & 2 \\
\hline & first quartile & 3 & 1 & 1 & 1 \\
\hline & third quartile & 7 & 4 & 5 & 3.5 \\
\hline \multirow[t]{4}{*}{ complex } & mean \pm SD & $1.3 \pm 1.1$ & $1.6 \pm 1.4$ & $1.5 \pm 1.3$ & $1.0 \pm 0.9$ \\
\hline & median & 1 & 1 & 1 & 1 \\
\hline & first quartile & 0.5 & 1 & 0.8 & 0 \\
\hline & third quartile & 2 & 2 & 2 & 1 \\
\hline \multirow[t]{4}{*}{$\mathrm{F}_{\mathrm{G}} / 100$} & mean $\pm \mathrm{SD}$ & $2.49 \pm 1.38$ & $2.25 \pm 1.24$ & $2.30 \pm 1.27$ & $1.84 \pm 1.12$ \\
\hline & median & 2.33 & 2.10 & 2.10 & 1.57 \\
\hline & first quartile & 1.17 & 1.31 & 1.31 & 1.04 \\
\hline & third quartile & 3.26 & 3.15 & 3.15 & 2.61 \\
\hline
\end{tabular}

Abbreviations: Ab.c., aberrant cells/1000 cells; rcp, reciprocal translocations/1000 cells; $t$, total number of translocations/1000 cells; dic, dicentric chromosomes/1000 cells; ace, acentric chromosomes/1000 cells; complex, cells with complex rearrangement (three or more breaks in two or more chromosomes)/1000 cells; $\mathrm{F}_{\mathrm{G}} / 100$, genomic frequency of stable translocations/100 cells.

$\mathrm{p}<0.001)$ and acentric fragments $(\mathrm{aOR}=1.36, \mathrm{p}<0.001)$ in patients with gastrointestinal cancer in comparison with controls. The same cytogenetic endpoints were elevated when gastrointestinal cancer patients were compared with breast cancer patients $(\mathrm{aOR}=1.10, \mathrm{p}=0.041 ; \mathrm{aOR}=1.85, \mathrm{p}<0.001$; $\mathrm{aOR}=1.30, \mathrm{p}=0.001)$. Breast cancer patients manifested an elevated frequency of cells with complex rearrangements compared with controls $(\mathrm{aOR}=1.46, \mathrm{p}=0.014)$. The groups of cancer patients did not differ from controls in any parameter regarding translocations.

Subsequently, subjects in the groups were divided according to their smoking habits (Tables 3 and 4 ) and the association of cytogenetic parameters with cancer development was evaluated in never smokers and ever smokers (current or former smokers) separately (Table 2). In regard to nonsmokers, a significant increase of aberrant cells $(\mathrm{aOR}=1.22, \mathrm{p}=0.016)$, dicentric chromosomes $(\mathrm{aOR}=3.80, \mathrm{p}=0.002)$, and acentric fragments $(\mathrm{aOR}=1.60, \mathrm{p}=0.002)$ were pronounced in the group of patients with gastrointestinal cancer when compared with controls. Elevated frequencies of dicentric chromosomes $(\mathrm{aOR}=1.93, \mathrm{p}=0.004)$ and acentric fragments $(\mathrm{aOR}=1.31, \mathrm{p}=0.007)$ were also observed in nonsmokers with gastrointestinal cancer when compared to nonsmokers with breast cancer. In nonsmokers with breast cancer, aside from the elevation of cells with complex rearrangements $(\mathrm{aOR}=1.93, \mathrm{p}=0.006)$, we also observed a significant increase in the frequency of total translocations $(\mathrm{aOR}=1.07, \mathrm{p}=0.035)$ and genomic frequency of stable translocations $(\mathrm{aOR}=1.63$, 
Table 2. Odds ratios of cytogenetic parameters for cancer patients (adjusted for age and smoking).

\begin{tabular}{|c|c|c|c|c|c|c|c|c|c|c|c|c|c|c|c|c|}
\hline \multirow{3}{*}{ All subjects } & \multicolumn{4}{|c|}{$\begin{array}{c}\text { Gastrointestinal cancer } \\
\text { (vs. controls) }\end{array}$} & \multicolumn{4}{|c|}{$\begin{array}{l}\text { Breast cancer } \\
\text { (vs. controls) }\end{array}$} & \multicolumn{4}{|c|}{$\begin{array}{l}\text { All cancers } \\
\text { (vs. controls) }\end{array}$} & \multicolumn{4}{|c|}{$\begin{array}{l}\text { Gastrointestinal cancer } \\
\text { vs. breast cancer }\end{array}$} \\
\hline & \multirow{2}{*}{ aOR } & \multicolumn{2}{|c|}{$95 \% \mathrm{CI}$} & \multirow{2}{*}{ p-value } & \multirow{2}{*}{ aOR } & \multicolumn{2}{|c|}{$95 \% \mathrm{CI}$} & \multirow{2}{*}{ p-value } & \multirow{2}{*}{ aOR } & \multicolumn{2}{|c|}{$95 \% \mathrm{CI}$} & \multirow{2}{*}{ p-value } & \multirow{2}{*}{ aOR } & \multicolumn{2}{|c|}{$95 \% \mathrm{CI}$} & \multirow{2}{*}{ p-value } \\
\hline & & lower & upper & & & lower & upper & & & lower & upper & & & lower & upper & \\
\hline Ab.c. & 1.16 & 1.04 & 1.30 & 0.009 & 1.05 & 0.97 & 1.14 & 0.215 & 1.08 & 1.00 & 1.16 & 0.056 & 1.10 & 1.00 & 1.21 & 0.041 \\
\hline $\mathrm{rcp}$ & 1.03 & 0.91 & 1.18 & 0.630 & 1.04 & 0.95 & 1.15 & 0.405 & 1.06 & 0.95 & 1.14 & 0.387 & 1.01 & 0.90 & 1.13 & 0.875 \\
\hline $\mathrm{t}$ & 1.03 & 0.97 & 1.09 & 0.321 & 1.03 & 0.98 & 1.07 & 0.231 & 1.14 & 0.98 & 1.31 & 0.066 & 1.01 & 0.96 & 1.07 & 0.653 \\
\hline dic & 2.70 & 1.64 & 4.45 & $<0.001$ & 1.14 & 0.84 & 1.54 & 0.395 & 1.36 & 1.31 & 1.80 & 0.030 & 1.85 & 1.31 & 2.61 & $<0.001$ \\
\hline ace & 1.36 & 1.15 & 1.62 & $<0.001$ & 1.04 & 0.91 & 1.18 & 0.575 & 1.11 & 0.98 & 1.24 & 0.084 & 1.30 & 1.12 & 1.52 & 0.001 \\
\hline complex & 1.24 & 0.78 & 1.97 & 0.375 & 1.46 & 1.08 & 1.98 & 0.014 & 1.38 & 1.03 & 1.84 & 0.029 & 0.78 & 0.54 & 1.14 & 0.196 \\
\hline $\mathrm{F}_{\mathrm{G}} / 100$ & 1.25 & 0.81 & 1.93 & 0.314 & 1.23 & 0.90 & 1.69 & 0.199 & 1.24 & 0.92 & 1.66 & 0.160 & 1.10 & 0.75 & 1.60 & 0.635 \\
\hline \multicolumn{17}{|l|}{ Nonsmokers } \\
\hline Ab.c. & 1.22 & 1.04 & 1.43 & 0.016 & 1.14 & 1.01 & 1.27 & 0.033 & 1.14 & 1.03 & 1.27 & 0.014 & 1.07 & 0.96 & 1.20 & 0.219 \\
\hline $\mathrm{rcp}$ & 1.12 & 0.92 & 1.36 & 0.272 & 1.12 & 0.97 & 1.30 & 0.111 & 1.12 & 0.98 & 1.27 & 0.097 & 1.01 & 0.87 & 1.17 & 0.889 \\
\hline $\mathrm{t}$ & 1.06 & 0.97 & 1.16 & 0.178 & 1.07 & 1.01 & 1.14 & 0.035 & 1.26 & 1.02 & 1.54 & 0.029 & 1.00 & 0.93 & 1.07 & 0.995 \\
\hline dic & 3.80 & 1.61 & 8.94 & 0.002 & 1.34 & 0.86 & 2.07 & 0.195 & 1.55 & 1.04 & 2.33 & 0.034 & 1.93 & 1.24 & 3.00 & 0.004 \\
\hline ace & 1.60 & 1.19 & 2.14 & 0.002 & 1.12 & 0.93 & 1.35 & 0.230 & 1.19 & 1.00 & 1.42 & 0.050 & 1.31 & 1.08 & 1.59 & 0.007 \\
\hline complex & 1.68 & 0.84 & 3.36 & 0.146 & 1.93 & 1.20 & 3.10 & 0.006 & 1.82 & 1.16 & 2.84 & 0.009 & 0.75 & 0.43 & 1.30 & 0.297 \\
\hline $\mathrm{F}_{\mathrm{G}} / 100$ & 1.57 & 0.82 & 2.97 & 0.171 & 1.63 & 1.03 & 2.58 & 0.038 & 1.60 & 1.04 & 2.47 & 0.032 & 1.04 & 0.64 & 1.68 & 0.888 \\
\hline \multicolumn{17}{|l|}{ Smokers } \\
\hline Ab.c. & 1.11 & 0.94 & 1.31 & 0.215 & 0.95 & 0.83 & 1.09 & 0.497 & 1.02 & 0.93 & 1.13 & 0.647 & 1.17 & 0.97 & 1.40 & 0.096 \\
\hline $\mathrm{rcp}$ & 0.97 & 0.80 & 1.17 & 0.728 & 0.98 & 0.85 & 1.13 & 0.759 & 1.00 & 0.89 & 1.13 & 0.959 & 1.00 & 0.82 & 1.21 & 0.966 \\
\hline $\mathrm{t}$ & 1.01 & 0.93 & 1.09 & 0.894 & 0.98 & 0.92 & 1.05 & 0.613 & 1.01 & 0.96 & 1.06 & 0.848 & 1.03 & 0.94 & 1.13 & 0.526 \\
\hline dic & 2.06 & 1.07 & 3.96 & 0.031 & 0.96 & 0.61 & 1.51 & 0.856 & 1.20 & 0.80 & 1.79 & 0.374 & 1.75 & 0.99 & 3.08 & 0.054 \\
\hline ace & 1.22 & 0.99 & 1.51 & 0.067 & 0.95 & 0.79 & 1.16 & 0.642 & 1.05 & 0.90 & 1.22 & 0.566 & 1.29 & 1.00 & 1.65 & 0.050 \\
\hline complex & 0.98 & 0.52 & 1.87 & 0.959 & 1.18 & 0.79 & 1.78 & 0.418 & 1.15 & 0.80 & 1.66 & 0.443 & 0.79 & 0.47 & 1.34 & 0.386 \\
\hline $\mathrm{F}_{\mathrm{G}} / 100$ & 1.03 & 0.56 & 1.89 & 0.930 & 0.90 & 0.55 & 1.48 & 0.679 & 0.94 & 0.60 & 1.49 & 0.803 & 1.17 & 0.62 & 2.22 & 0.623 \\
\hline
\end{tabular}

Abbreviations: Ab.c., aberrant cells/1000 cells; rcp, reciprocal translocations/1000 cells; t, total number of translocations/1000 cells; dic, dicentric chromosomes/1000 cells; ace, acentric chromosomes/1000 cells; complex, cells with complex rearrangement (three or more breaks in two or more chromosomes)/1000 cells; $\mathrm{F}_{\mathrm{G}} / 100$, genomic frequency of stable translocations/100 cells; aOR, adjusted odds ratio; CI, confidence interval; significant values are in bold $(\mathrm{p}<0.05)$

$\mathrm{p}=0.038$ ) that was not seen when all breast cancer patients (smokers and nonsmokers) were compared with controls. In smokers, only dicentric chromosomes $(\mathrm{aOR}=2.06, \mathrm{p}=0.031)$ were elevated and acentric fragments were near the statistical significance $(\mathrm{aOR}=1.22, \mathrm{p}=0.067)$ in gastrointestinal cancer patients when compared to controls. No differences in cytogenetic endpoints were observed between smoker breast cancer patients and controls.

Furthermore, we compared cytogenetic parameters between smokers and nonsmokers in groups of patients and controls (Table 5). Among control subjects, smokers had significantly higher frequencies of all examined cytogenetic parameters except dicentric chromosomes. However, smoking status did not affect cytogenetic parameters in groups of newly diagnosed cancer patients.

We also compared the cytogenetic parameters with TNM stages. For statistical analysis, gastrointestinal cancer patients were divided into two groups with TNM I + II and TNM III + IV, and breast cancer patients into groups with TNM I and TNM II + III. We did not reveal an association between evaluated cytogenetic parameters and TNM stages except for unstable dicentric chromosomes in gastrointestinal cancer patients, where higher TNM stages were associated with a lower frequency of dicentric chromosomes $(\mathrm{aOR}=0.40$, $\mathrm{p}=0.033$ ).

\section{Discussion}

Breast and colorectal cancer rank among the most frequently diagnosed cancers and are the leading causes of cancer death in developed countries [22]. In our study, we focused on chromosomal damage detected by FISH with painting probes in patients with newly diagnosed cancer and on the impact of tobacco smoke exposure on this association. As we demonstrated in nonsmokers, untreated gastrointestinal and breast cancer patients had an increased frequency of aberrant cells. However, chromosome damage affected different cytogenetic endpoints in these groups. Gastrointestinal cancer is associated with elevated frequency of unstable chromosome aberrations such as dicentric chromosomes and acentric fragments. In contrast, stable translocations and cells with complex rearrangements contributed to chromosome damage in breast cancer. Only a few studies dealing with this topic have been published. Increased levels of aberrant 
Table 3. Chromosomal aberrations in newly diagnosed cancer patients and controls (nonsmokers).

\begin{tabular}{|c|c|c|c|c|c|}
\hline \multirow{2}{*}{\multicolumn{2}{|c|}{ Nonsmokers }} & \multicolumn{3}{|c|}{ Cancer patients } & \multirow[b]{2}{*}{$\begin{array}{c}\text { Controls } \\
\mathrm{N}=44\end{array}$} \\
\hline & & \multirow{2}{*}{$\begin{array}{c}\begin{array}{c}\text { Gastrointestinal } \\
\mathbf{N}=\mathbf{1 3}\end{array} \\
61.5 \pm 9.1\end{array}$} & \multirow{2}{*}{$\begin{array}{c}\begin{array}{c}\text { Breast } \\
\mathrm{N}=67\end{array} \\
61.2 \pm 12.2\end{array}$} & \multirow{2}{*}{$\begin{array}{c}\begin{array}{c}\text { Total } \\
\mathbf{N}=\mathbf{8 0}\end{array} \\
61.2 \pm 11.7\end{array}$} & \\
\hline Age & mean $\pm S D$ & & & & $53.1 \pm 13.4$ \\
\hline \multirow[t]{3}{*}{ (years) } & median & 63.9 & 62.6 & 63.0 & 51.9 \\
\hline & first quartile & 52.9 & 51.2 & 51.5 & 44.7 \\
\hline & third quartile & 69.0 & 68.6 & 68.9 & 63.0 \\
\hline \multirow[t]{4}{*}{ Ab.c. } & mean $\pm \mathrm{SD}$ & $12.3 \pm 6.4$ & $10.3 \pm 5.2$ & $10.7 \pm 5.5$ & $7.2 \pm 3.6$ \\
\hline & median & 14 & 10 & 10.5 & 7 \\
\hline & first quartile & 6 & 7 & 6 & 4 \\
\hline & third quartile & 16 & 13 & 14 & 9.25 \\
\hline \multirow[t]{4}{*}{ rcp } & mean $\pm \mathrm{SD}$ & $6.8 \pm 5.0$ & $6.6 \pm 4.1$ & $6.6 \pm 4.2$ & $4.5 \pm 2.8$ \\
\hline & median & 6 & 5 & 5 & 4 \\
\hline & first quartile & 3 & 4 & 4 & 2 \\
\hline & third quartile & 10 & 8.5 & 9 & 6 \\
\hline \multirow[t]{4}{*}{$\mathrm{t}$} & mean $\pm S D$ & $16.7 \pm 12.0$ & $16.6 \pm 9.3$ & $16.6 \pm 9.5$ & $10.9 \pm 6.6$ \\
\hline & median & 18 & 14 & 14 & 9 \\
\hline & first quartile & 8 & 9 & 9 & 5.75 \\
\hline & third quartile & 23 & 22 & 22 & 14 \\
\hline \multirow[t]{4}{*}{ dic } & mean $\pm \mathrm{SD}$ & $2.5 \pm 1.7$ & $1.1 \pm 1.2$ & $1.3 \pm 1.4$ & $0.7 \pm 0.8$ \\
\hline & median & 2 & 1 & 1 & 1 \\
\hline & first quartile & 1 & 0 & 0 & 0 \\
\hline & third quartile & 3 & 2 & 2 & 1 \\
\hline \multirow[t]{4}{*}{ ace } & mean $\pm S D$ & $5.4 \pm 3.5$ & $2.8 \pm 2.7$ & $3.2 \pm 2.9$ & $2.1 \pm 1.9$ \\
\hline & median & 4 & 2 & 3 & 2 \\
\hline & first quartile & 3 & 1 & 1 & 0.75 \\
\hline & third quartile & 7 & 4 & 4.2 & 3 \\
\hline \multirow[t]{4}{*}{ complex } & mean $\pm \mathrm{SD}$ & $1.2 \pm 1.1$ & $1.5 \pm 1.3$ & $1.5 \pm 1.3$ & $0.8 \pm 0.8$ \\
\hline & median & 1 & 1 & 1 & 1 \\
\hline & first quartile & 0 & 1 & 0.8 & 0 \\
\hline & third quartile & 2 & 2 & 2 & 1 \\
\hline \multirow[t]{4}{*}{$\mathrm{F}_{\mathrm{G}} / 100$} & mean $\pm \mathrm{SD}$ & $2.31 \pm 1.48$ & $2.24 \pm 1.27$ & $2.25 \pm 1.30$ & $1.49 \pm 0.91$ \\
\hline & median & 2.33 & 1.84 & 1.97 & 1.30 \\
\hline & first quartile & 1.05 & 1.31 & 1.31 & 0.79 \\
\hline & third quartile & 3.15 & 3.15 & 3.15 & 1.84 \\
\hline
\end{tabular}

Abbreviations: Ab.c., aberrant cells/1000 cells; rcp, reciprocal translocations/1000 cells; t, total number of translocations/1000 cells; dic, dicentric chromosomes/1000 cells; ace, acentric chromosomes/1000 cells; complex, cells with complex rearrangement (three or more breaks in two or more chromosomes)/1000 cells; $\mathrm{F}_{\mathrm{G}} / 100$, genomic frequency of stable translocations/100 cells.

cells with unstable chromosome aberrations detected by conventional analysis using Giemsa stained chromosomes were found in untreated breast, prostate, uterus/ovary, head/neck, bladder/kidney and lung cancer patients [7, 23]. As the authors did not observe increased levels of typical postradiation aberrations (i.e., dicentric chromosomes), they concluded that more frequent diagnostic X-ray examination during diagnosis in these patients did not have a critical effect. Another study showed an increase in conventional cytogenetic endpoints including dicentric chromosomes in lung cancer patients [8]. In our study, the level of dicentric chromosomes in gastrointestinal cancer patients was elevated in both nonsmokers and smokers. Thus, it cannot be ruled out that acute exposure to diagnostic X-rays might have contributed to the increase of unstable aberrations found here. No elevation of the frequency of dicentric chromosomes was observed in breast cancer patients. In fact, gastrointestinal cancer patients receive greater radiation doses during diagnostic examinations compared to breast cancer patients (median $29.83 \mathrm{mSv}$ vs. $0.35 \mathrm{mSv}$, unpublished results of MMCI) because they routinely undergo computed tomography scans.

Contrary to findings in gastrointestinal cancer patients, an increased level of stable translocations was observed in breast cancer patients (nonsmokers). Our finding is in agreement with the study by Verdorfer et al. [4] who investigated 
Table 4. Chromosomal aberrations in newly diagnosed cancer patients and controls (smokers).

\begin{tabular}{|c|c|c|c|c|c|}
\hline \multirow{2}{*}{ Smokers } & & \multicolumn{3}{|c|}{ Cancer patients } & \multirow[b]{2}{*}{$\begin{array}{c}\text { Controls } \\
\mathrm{N}=35(8 / 27)^{*}\end{array}$} \\
\hline & & $\begin{array}{c}\text { Gastrointestinal } \\
\mathrm{N}=14(3 / 11)^{*}\end{array}$ & $\begin{array}{c}\text { Breast } \\
\mathrm{N}=30(14 / 16)^{*}\end{array}$ & $\begin{array}{c}\text { Total } \\
\mathrm{N}=44(17 / 27)^{*}\end{array}$ & \\
\hline Age & mean $\pm S D$ & $62.3 \pm 10.7$ & $58.6 \pm 12.6$ & $59.8 \pm 12.0$ & $56.8 \pm 12.1$ \\
\hline \multirow[t]{3}{*}{ (years) } & median & 63.3 & 60.7 & 62.5 & 60.2 \\
\hline & first quartile & 57.1 & 50.8 & 51.5 & 50.0 \\
\hline & third quartile & 68.1 & 68.3 & 68.7 & 66.3 \\
\hline \multirow[t]{4}{*}{ Ab.c. } & mean $\pm S D$ & $13.6 \pm 5.8$ & $10.3 \pm 4.7$ & $11.4 \pm 5.2$ & $10.5 \pm 4.6$ \\
\hline & median & 14 & 9.5 & 10.5 & 10 \\
\hline & first quartile & 10.25 & 7.25 & 8 & 7.5 \\
\hline & third quartile & 17 & 14 & 15.3 & 13.5 \\
\hline \multirow[t]{4}{*}{$\mathrm{rcp}$} & mean $\pm S D$ & $7.6 \pm 4.0$ & $7.0 \pm 4.1$ & $7.2 \pm 4.0$ & $6.9 \pm 4.3$ \\
\hline & median & 7.5 & 6 & 6.5 & 7 \\
\hline & first quartile & 4.25 & 4 & 4 & 4 \\
\hline & third quartile & 11 & 10 & 10.3 & 9 \\
\hline \multirow[t]{4}{*}{$\mathrm{t}$} & mean $\pm S D$ & $19.4 \pm 9.6$ & $16.2 \pm 8.7$ & $17.2 \pm 9.0$ & $16.4 \pm 9.4$ \\
\hline & median & 18.5 & 15 & 16 & 14 \\
\hline & first quartile & 15 & 8.5 & 9.8 & 10 \\
\hline & third quartile & 25.5 & 22 & 24.3 & 22.5 \\
\hline \multirow[t]{4}{*}{ dic } & mean $\pm \mathrm{SD}$ & $1.7 \pm 1.1$ & $0.9 \pm 1.2$ & $1.1 \pm 1.2$ & $0.9 \pm 1.0$ \\
\hline & median & 2 & 0 & 1 & 1 \\
\hline & first quartile & 1.25 & 0 & 0 & 0 \\
\hline & third quartile & 2 & 1 & 2 & 1.5 \\
\hline \multirow[t]{4}{*}{ ace } & mean $\pm S D$ & $5.3 \pm 3.8$ & $2.9 \pm 2.5$ & $3.7 \pm 3.1$ & $3.2 \pm 2.7$ \\
\hline & median & 4 & 2 & 3 & 3 \\
\hline & first quartile & 3 & 1 & 1 & 1 \\
\hline & third quartile & 5.75 & 4 & 5.3 & 4 \\
\hline \multirow[t]{4}{*}{ complex } & mean $\pm S D$ & $1.4 \pm 1.1$ & $1.6 \pm 1.6$ & $1.6 \pm 1.5$ & $1.31 \pm 1.0$ \\
\hline & median & 1 & 1 & 1 & 1 \\
\hline & first quartile & 1 & 0 & 0.8 & 1 \\
\hline & third quartile & 2 & 2.75 & 2 & 2 \\
\hline \multirow[t]{4}{*}{$\mathrm{F}_{\mathrm{G}} / 100$} & mean $\pm \mathrm{SD}$ & $2.65 \pm 1.32$ & $2.27 \pm 1.18$ & $2.40 \pm 1.23$ & $2.28 \pm 1.22$ \\
\hline & median & 2.33 & 2.10 & 2.33 & 1.84 \\
\hline & first quartile & 1.90 & 1.38 & 1.51 & 1.43 \\
\hline & third quartile & 3.70 & 3.08 & 3.15 & 3.00 \\
\hline
\end{tabular}

* number of current/former smokers in parenthesis

Abbreviations: Ab.c., aberrant cells/1000 cells; rcp, reciprocal translocations/1000 cells; t, total number of translocations/1000 cells; dic, dicentric chromosomes/1000 cells; ace, acentric chromosomes/1000 cells; complex, cells with complex rearrangement (three or more breaks in two or more chromosomes)/1000 cells; $\mathrm{F}_{\mathrm{G}} / 100$, genomic frequency of stable translocations/100 cells.

32 breast cancer patients by FISH. Stable aberrations essentially contributed to the increased number of aberrant cells detected in their study.

The advantage of FISH over conventional analysis is the ability to detect stable aberrations. However, FISH is also able to detect unstable aberrations represented by dicentric chromosomes and acentric fragments. Though the results are limited to the fraction of the genome painted by FISH, more cells are commonly evaluated by this method than by a conventional one. Fluorescence staining is less appropriate for visualization of chromosome breaks and, therefore, these aberrations were not evaluated by FISH.
The case-control design of this study is not appropriate to discern if chromosomal aberrations are a biomarker for cancer risk or are the consequence of disease progression. However, large cohort studies substantiate the use of chromosome aberrations as an early predictive biomarker. Chromosome aberration frequency appears to be particularly associated with gastrointestinal cancer, mainly for stomach cancer [2, 3, 24]. For breast cancer, the results were only suggestive of an association. As these prospective studies evaluated unstable aberrations using conventionally stained chromosomes, the sensitivity of the biomarker can be compromised. Stable aberrations, which tend to accumulate 
Table 5. Odds ratios of cytogenetic parameters for smokers in comparison with nonsmokers (adjusted for age).

\begin{tabular}{|c|c|c|c|c|c|c|c|c|c|c|c|c|c|c|c|c|}
\hline & \multicolumn{4}{|c|}{ Controls } & \multicolumn{4}{|c|}{ Gastrointestinal cancer } & \multicolumn{4}{|c|}{ Breast cancer } & \multicolumn{4}{|c|}{ All cancers } \\
\hline & \multirow{2}{*}{ aOR } & \multicolumn{2}{|c|}{$95 \%$ CI } & \multirow{2}{*}{ p-value } & \multirow{2}{*}{ aOR } & \multicolumn{2}{|c|}{$95 \%$ CI } & \multirow{2}{*}{ p-value } & \multirow{2}{*}{ aOR } & \multicolumn{2}{|c|}{$95 \%$ CI } & \multirow{2}{*}{ p-value } & \multirow{2}{*}{ aOR } & \multicolumn{2}{|c|}{$95 \% \mathrm{CI}$} & \multirow{2}{*}{ p-value } \\
\hline & & lower & upper & & & lower & upper & & & lower & upper & & & lower & upper & \\
\hline Ab.c. & 1.27 & 1.09 & 1.48 & 0.002 & 1.04 & 0.90 & 1.19 & 0.588 & 1.03 & 0.93 & 1.14 & 0.591 & 1.05 & 0.97 & 1.13 & 0.251 \\
\hline $\mathrm{rcp}$ & 1.24 & 1.04 & 1.48 & 0.014 & 1.05 & 0.87 & 1.26 & 0.636 & 1.06 & 0.94 & 1.20 & 0.325 & 1.06 & 0.96 & 1.17 & 0.260 \\
\hline $\mathrm{t}$ & 1.10 & 1.02 & 1.19 & 0.012 & 1.03 & 0.95 & 1.12 & 0.513 & 1.01 & 0.95 & 1.07 & 0.752 & 0.92 & 0.78 & 1.08 & 0.315 \\
\hline dic & 1.20 & 0.73 & 1.98 & 0.468 & 0.66 & 0.35 & 1.23 & 0.190 & 0.87 & 0.58 & 1.30 & 0.494 & 0.90 & 0.67 & 1.22 & 0.505 \\
\hline ace & 1.26 & 1.02 & 1.55 & 0.035 & 0.99 & 0.80 & 1.23 & 0.918 & 1.05 & 0.89 & 1.25 & 0.560 & 1.07 & 0.94 & 1.21 & 0.289 \\
\hline complex & 2.01 & 1.16 & 3.49 & 0.013 & 1.26 & 0.61 & 2.62 & 0.535 & 1.15 & 0.82 & 1.61 & 0.429 & 1.11 & 0.83 & 1.49 & 0.487 \\
\hline $\mathrm{F}_{\mathrm{G}} / 100$ & 2.29 & 1.27 & 4.13 & 0.006 & 1.22 & 0.65 & 2.28 & 0.533 & 1.13 & 0.76 & 1.67 & 0.558 & 0.33 & 1.17 & 0.85 & 1.624 \\
\hline
\end{tabular}

Abbreviations: Ab.c., aberrant cells/1000 cells; rcp, reciprocal translocations/1000 cells; t, total number of translocations/1000 cells; dic, dicentric chromosomes/1000 cells; ace, acentric chromosomes/1000 cells; complex, cells with complex rearrangement (three or more breaks in two or more chromosomes)/1000 cells; $\mathrm{F}_{\mathrm{G}} / 100$, genomic frequency of stable translocations/100 cells; aOR, adjusted odds ratio; CI, confidence interval; significant values are in bold $(\mathrm{p}<0.05)$

in the organism, may more reliably reflect increased susceptibility to chromosomal damage. Thus, translocations detected by chromosome painting may provide a cancer risk estimate superior to the traditional chromosome aberration analysis. In our study, we demonstrated that increased levels of stable translocations are associated with breast cancer. No association was revealed between stable cytogenetic parameters and TNM stages in any patient group. However, a limited number of patients, especially in the group of gastrointestinal cancer and prevailing lower stages in breast cancer patients, did not enable us to reach an undisputed conclusion on this topic. As regards unstable aberrations, a lower level of dicentric chromosomes in advanced TNM stages in our gastrointestinal cancer patients could be influenced by different exposure to X-rays between TNM groups during the diagnostic examination.

Tobacco smoke is one of the most important carcinogens and thus smoking is an established risk factor associated with many cancers, including gastrointestinal cancer [22]. It has been shown that cigarette smoking increases chromosome aberrations [25-28]. This is in agreement with our observation in healthy controls where chronic exposure to tobacco smoke increased the frequencies of stable and unstable chromosome aberrations except the dicentric chromosomes. However, this smoking related difference was not observed in groups of cancer patients. Furthermore, an association between chromosome damage and cancer observed in nonsmokers was unpronounced in smokers (with the exception of dicentric chromosomes in gastrointestinal patients). A similar effect of exposure to tobacco smoke and environmental pollution on chromosome aberrations was detected in other studies $[8,15]$. It has been postulated that chronic exposure to environmental stressors can lead to adaptation of organisms via epigenetic mechanisms, which helps to reduce additional DNA damage [29]. It is well documented that genome methylation is strongly affected by smoking habits. Some sites remain differentially methylated for several decades after smoking cessation [30-33]. Thus, adaptation to chronic exposure of tobacco smoke in former and current smokers probably helped to reduce the level of additional DNA damage in our cancer patients.

Different types of chromosome damage were observed in two groups of untreated cancer patients using FISH method with whole chromosome painting probes. Increased frequency of unstable aberrations detected in gastrointestinal cancer patients can be at least partly ascribed to acute exposure to diagnostic X-rays. In contrast, stable rearrangements that can persist in the body and cells with complex rearrangements contributed to chromosome damage in breast cancer patients.

Acknowledgements: Supported by Ministry of Health of the Czech Republic, grant no. 15-33968A.

\section{References}

[1] ROSSNER P, BOFFETTA P, CEPPI M, BONASSI S, SMERHOVSKY Z et al. Chromosomal Aberrations in Lymphocytes of Healthy Subjects and Risk of Cancer. Environ Health Perspect 2005; 113: 517-520. https://doi.org/10.1289/ehp.6925

[2] NORPPA H, BONASSI S, HANSTEEN I-L, HAGMAR L, STRÖMBERG U et al. Chromosomal aberrations and SCEs as biomarkers of cancer risk. Mutat Res 2006; 600: 37-45. https://doi.org/10.1016/j.mrfmmm.2006.05.030

[3] BONASSI S, NORPPA H, CEPPI M, STROMBERG U, VERMEULEN R et al. aberration frequency in lymphocytes predicts the risk of cancer: results from a pooled cohort study of 22358 subjects in 11 countries. Carcinogenesis 2008; 29: 1178-1183. https://doi.org/10.1093/carcin/bgn075

[4] VERDORFER I, NEUBAUER S, LETZEL S, ANGERER J, ARUTYUNYAN R et al. Chromosome painting for cytogenetic monitoring of occupationally exposed and nonexposed groups of human individuals. Mutat Res 2001; 491: 97-109. https://doi.org/10.1016/s1383-5718(01)00128-0

[5] M'KACHER R, GIRINSKY T, KOSCIELNY S, DOSSOU J, VIOLOT D et al. Baseline and treatment-induced chromosomal abnormalities in peripheral blood lymphocytes of Hodgkin's lymphoma patients. Int J Radiat Oncol Biol Phys 2003; 57: 321-326. https://doi.org/10.1016/s03603016(03)00578-9 
[6] MAFFEI F, MORAGA Z, MANUEL J, ANGELINI S, ZENESINI $C$ et al. Micronucleus frequency in human peripheral blood lymphocytes as a biomarker for the early detection of colorectal cancer risk. Mutagenesis 2014; 29: 221-225. https://doi.org/10.1093/mutage/geu007

[7] VODENKOVA S, POLIVKOVA Z, MUSAK L, SMERHOVSKY Z, ZOUBKOVA H et al. Structural chromosomal aberrations as potential risk markers in incident cancer patients. Mutagenesis 2015; 30: 557-563. https://doi. org/10.1093/mutage/gev018

[8] MININA VI, SINITSKY MY, DRUZHININ VG, FUCIC A, BAKANOVA ML et al. Chromosome aberrations in peripheral blood lymphocytes of lung cancer patients exposed to radon and air pollution. Eur J Cancer Prev 2018; 27: 6-12. https://doi.org/10.1097/CEJ.0000000000000270

[9] GARCIA-SAGREDO JM. Fifty years of cytogenetics: A parallel view of the evolution of cytogenetics and genotoxicology. Biochim Biophys Acta 2008; 1779: 363-375. https://doi. org/10.1016/j.bbagrm.2008.05.003

[10] KADDOUR A, COLICCHIO B, BURON D, EL MAALOUF E, LAPLAGNE E et al. Transmission of Induced Chromosomal Aberrations through Successive Mitotic Divisions in Human Lymphocytes after In Vitro and In Vivo Radiation. Sci Rep 2017; 7: 3291. https://doi.org/10.1038/s41598-01703198-7

[11] INTERNATIONAL ATOMIC ENERGY AGENCY. Cytogenetic Dosimetry: Applications in Preparedness for and Response to Radiation Emergencies. IAEA, Vienna 2011.

[12] PERNOT E, HALL J, BAATOUT S, BENOTMANE MA, BLANCHARDON E et al. Ionizing radiation biomarkers for potential use in epidemiological studies. Mutat Res 2012; 751: 258-286. https://doi.org/10.1016/j.mrrev.2012.05.003

[13] GRÉGOIRE E, ROY L, BUARD V, DELBOS M, DURAND V et al. Twenty years of FISH-based translocation analysis for retrospective ionizing radiation biodosimetry. Int J Radiat Biol 2018; 94: 248-258. https://doi.org/10.1080/09553002.2 018.1427903

[14] SRAM RJ, BESKID O, BINKOVA B, ROSSNER P, SMERHOVSKY Z. Cytogenetic analysis using fluorescence in situ hybridization (FISH) to evaluate occupational exposure to carcinogens. Toxicol Lett 2004; 149: 335-344. https://doi. org/10.1016/j.toxlet.2003.12.043

[15] SRAM RJ, BESKID O, BINKOVA B, CHVATALOVA I, LNENICKOVA $Z$ et al. Chromosomal aberrations in environmentally exposed population in relation to metabolic and DNA repair genes polymorphisms. Mutat Res 2007; 620: 22-33. https://doi.org/10.1016/j.mrfmmm.2007.02.019

[16] SRAM RJ, BESKID O, ROESSNEROVA A, ROESSNER P, LNENICKOVA $\mathrm{Z}$ et al. Environmental exposure to carcinogenic polycyclic aromatic hydrocarbons - The interpretation of cytogenetic analysis by FISH. Toxicol Lett 2007; 172: 12-20. https://doi.org/10.1016/j.toxlet.2007.05.019

[17] RUBES J, KUCHAROVÁ S, VOZDOVÁ M, MUSILOVÁ P, ZUDOVÁ Z. Cytogenetic analysis of peripheral lymphocytes in medical personnel by means of FISH. Mutat Res 1998; 412: 293-298. https://doi.org/10.1016/s1383-5718(97)00201-5
[18] TUCKER JD, MORGAN WF, AWA AA, BAUCHINGER M, BLAKEY D et al. A proposed system for scoring structural aberrations detected by chromosome painting. Cytogenet Cell Genet 1995; 68: 211-221. https://doi.org/10.1159/000133916 [19] POUZOULET F, ROCH-LEFÈVE S, GIRAUDET AL, VAURIJOUX A, VOISIN $P$ et al. Monitoring translocations by M-FISH and three-color FISH painting techniques: a study of two radiotherapy patients. J Radiat Res 2007; 48: 425-434. https://doi.org/10.1269/jrr.07013

[20] ABE Y, YOSHIDA MA, FUJIOKA K, KUROSU Y, UJIIE R et al. Dose-response curves for analyzing of dicentric chromosomes and chromosome translocations following doses of $1000 \mathrm{mGy}$ or less, based on irradiated peripheral blood samples from five healthy individuals. J Radiat Res 2018; 59: 35-42. https://doi.org/10.1093/jrr/rrx052

[21] LUCAS JN, SACHS RK. Using three-color chromosome painting to test chromosome aberration models. Proc Natl Acad Sci 1993; 90: 1484-1487.

[22] TORRE LA, BRAY F, SIEGEL RL, FERLAY J, LORTETTIEULENT J et al. Global cancer statistics, 2012. CA Cancer J Clin 2015; 65: 87-108. https://doi.org/10.3322/caac.21262

[23] VODICKA P, POLIVKOVA Z, SYTAROVA S, DEMOVA $\mathrm{H}$, KUCEROVA $\mathrm{M}$ et al. Chromosomal damage in peripheral blood lymphocytes of newly diagnosed cancer patients and healthy controls. Carcinogenesis 2010; 31: 1238-1241. https://doi.org/10.1093/carcin/bgq056

[24] BOFFETTA P, VAN DER HEL O, NORPPA H, FABIANOVA E, FUCIC A et al. Chromosomal aberrations and cancer risk: Results of a cohort study from central europe. Am J Epidemiol 2007; 165: 36-43. https://doi.org/10.1093/aje/kwj367

[25] SINUÉS B, IZQUIERDO M, PEREZ VIGUERA J. Chromosome aberrations and urinary thioethers in smokers. $\mathrm{Mu}-$ tat Res 1990; 240: 289-293. https://doi.org/10.1016/01651218(90)90079-h

[26] SIGURDSON AJ, HA M, HAUPTMANN M, BHATTI P, SRAM RJ et al. International study of factors affecting human chromosome translocations. Mutat Res 2008; 652: 112121. https://doi.org/10.1016/j.mrgentox.2008.01.005

[27] BALACHANDAR V, KUMAR BL, SURESH K, SASIKALA K. Evaluation of Chromosome Aberrations in Subjects Exposed to Environmental Tobacco Smoke in Tamilnadu, India. Bull Environ Contam Toxicol 2008; 81: 270-276. https:// doi.org/10.1007/s00128-008-9489-3

[28] GINZKEY C, FRIEHS G, KOEHLER C, HACKENBERG S, HAGEN R et al. Assessment of nicotine-induced DNA damage in a genotoxicological test battery. Mutat Res 2013; 751: 34-39. https://doi.org/10.1016/j.mrgentox.2012.11.004

[29] ROSSNEROVA A, POKORNA M, SVECOVA V, SRAM RJ, TOPINKA J et al. Adaptation of the human population to the environment: Current knowledge, clues from Czech cytogenetic and "omics" biomonitoring studies and possible mechanisms. Mutat Res 2017; 773: 188-203. https://doi. org/10.1016/j.mrrev.2017.07.002

[30] GUIDA F, SANDANGER TM, CASTAGNE R, CAMPANELLA G, POLIDORO $S$ et al. Dynamics of smoking-induced genome-wide methylation changes with time since smoking cessation. Hum Mol Genet 2015; 24: 2349-2359. https://doi. org/10.1093/hmg/ddu751 
[31] JOEHANES R, JUST AC, MARIONI RE, PILLING LC, REYNOLDS LM et al. Epigenetic Signatures of Cigarette Smoking. Circ Cardiovasc Genet 2016; 9: 436-447. https:// doi.org/10.1161/CIRCGENETICS.116.001506

[32] VINEIS P, CHATZIIOANNOU A, CUNLIFFE VT, FLANAGAN JM, HANSON M et al. Epigenetic memory in response to environmental stressors. FASEB J 2017; 31: 2241-2251. https://doi.org/10.1096/fj.201601059RR
[33] WILSON R, WAHL S, PFEIFFER L, WARD-CAVINESS CK, KUNZE $S$ et al. The dynamics of smoking-related disturbed methylation: a two time-point study of methylation change in smokers, non-smokers and former smokers. BMC Genomics 2017; 18: 805. https://doi.org/10.1186/s12864-0174198-0 